\title{
Comment on "Characterizing interwell connectivity in waterflooded reservoirs using data-driven and reduced-physics models: a comparative study" by E. Artun DOI 10.1007/s00521-015-2152-0
}

\author{
Jerry L. Jensen ${ }^{1}$
}

Published online: 23 August 2016

(C) The Natural Computing Applications Forum 2016

The paper sets out to compare interwell connectivities estimated by three methods: reservoir flow simulation, artificial neural network (ANN), and the capacitance model (CRM). The author concludes (Table 4) that the ANN and CRM methods behave similarly when compared to the simulation-based results, but that the CRM gives slightly weaker performance.

The paper, however, raises some questions surrounding these conclusions.

1. The CRM connectivities listed in Table 5 do not appear to have been properly calculated. Based on the simulation model description, each injector well's CRM connectivities should sum to 1 , because the simulation model is a closed system and no fluid can leak in or out of the model. This requirement is clearly described in Yousef et al. [5]. For example, the connectivities listed in Table 5 for injector 1 sum to 1.75 , which is much different than 1 .

2. A similar analysis for the simulation-based connectivities also shows the Table 5 connectivities do not sum to 1 . However, in this case, the paper does not indicate how these connectivities are calculated.

3. If one assumes that the CRM and simulation-based connectivities listed in Table 5 can be normalized to 1 for each injector and compared, the plot below results. This shows a very different result than listed in

This comment refers to the article available at doi: 10.1007/s00521-015-2152-0.

Jerry L. Jensen

jjensen@ucalgary.ca

1 Department of Chemical and Petroleum Engineering, University of Calgary, Calgary, AB T2N 1N4, Canada
Table 4. The CRM connectivities agree much better with the simulation-based connectivities than the ANN values do. In particular, the ANN especially overpredicts connectivities for the better-connected well pairs.

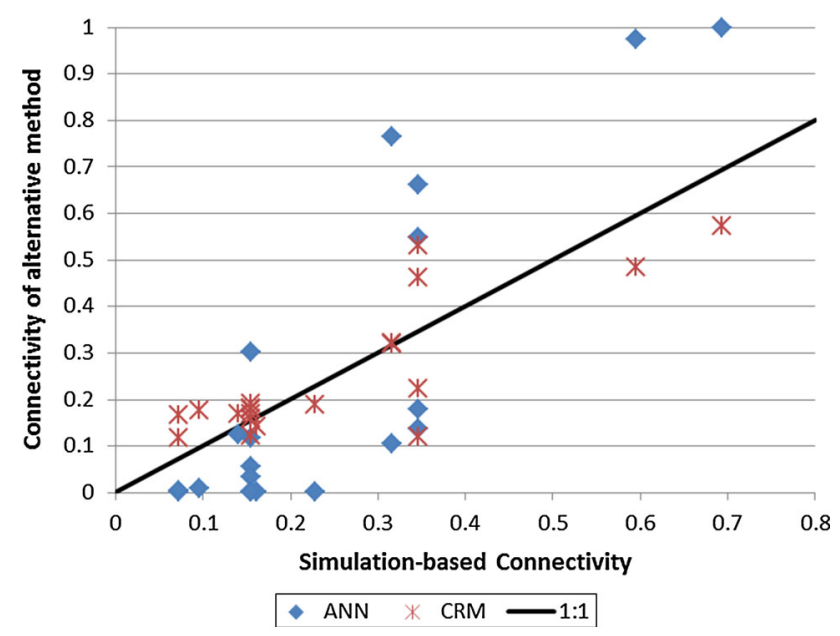

4. Two larger questions unaddressed in the paper are

(a) whether the connectivities of different methods are actually comparable. The CRM defines connectivity as the effect one unit of water injected has on a given producer. This is an open question for the ANN and simulation-based methods. (In the simulation-based case, this is because the paper does not give details on how the connectivities are evaluated.) Connectivity has been defined in a number of ways (e.g., $[1,2,6])$, and comparisons need to be undertaken with careful attention to what is meant by connectivity. 
(b) an advantage to the CRM approach to connectivity assessment is that there are clear assumptions and physics in the model. Therefore, the effects of common field disturbances such as temporarily closing a well or recompleting a producer can be explicitly identified and the model modified to make it more robust to these non-reservoir effects (e.g., [3, 4]). It is unclear how the ANN will perform under these circumstances.

Thus, while the study described undertakes an interesting comparison of methods to assess interwell connectivity, it requires more detail and a reassessment of the results.

\section{References}

1. Hovadik JM, Larue DK (2006) Connectivity of channelized reservoirs: a modelling approach. Pet Geosci 12:291-308

2. Kaviani D, Valkó PP (2010) Inferring interwell connectivity using Multiwell Productivity Index. J Pet Sci Eng 73:48-58
3. Kaviani D, Jensen JL, Lake LW (2012) Estimation of interwell connectivity in the case of fluctuating bottomhole pressures. J Petrol Sci Eng 90-91:79-95

4. Soroush M, Kaviani D, Jensen JL (2014) Interwell connectivity evaluation in cases of changing skin and frequent production interruptions. J Pet Sci Eng 122:616-630

5. Yousef AA, Gentil P, Jensen JL, Lake LW (2006) A capacitance model to infer interwell connectivity from production and injection rate fluctuations. SPEREE 9:630-646

6. Zhen Y, MacBeth C, Chassagne R (2015) Joint interpretation of interwell connectivity by integrating 4D seismic with injection and production fluctuations. Paper SPE 174365 presented at the Europec 2015 conference, Madrid, 1-4 June, p 13 